\title{
Der Innovationsfonds - neue Chance für eine bessere Versorgung schwer psychisch Kranker?
}

\author{
Funding for Health Care Innovation in Germany - New Chances for Optimized \\ Care for Severely Mentally III?
}

Autoren

Institute
Steffi G. Riedel-Heller ${ }^{1}$, Birgit Watzke², Martin Härter ${ }^{3}$, Hans-Helmut König ${ }^{4}$

${ }^{1}$ Institut für Sozialmedizin, Arbeitsmedizin und Public Health (ISAP), Medizinische Fakultät, Universität Leipzig

2 Psychologisches Institut, Lehrstuhl Klinische Psychologie und Psychotherapieforschung, Universität Zürich, Schweiz

${ }^{3}$ Institut und Poliklinik für Medizinische Psychologie, Universitätsklinikum Hamburg-Eppendorf

${ }^{4}$ Institut für Gesundheitsökonomie und Versorgungsforschung, Universitätsklinikum Hamburg-Eppendorf

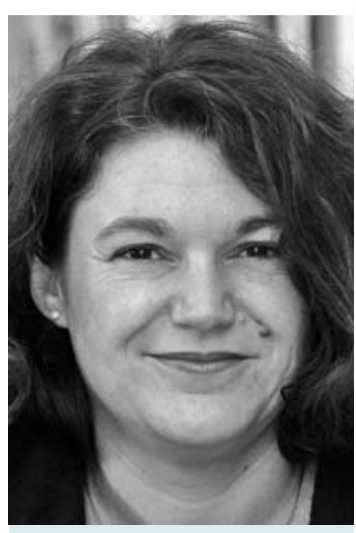

Steffi G. Riedel-Heller

Bibliografie

Dol http://dx.doi.org/

$10.1055 / \mathrm{s}-0035-1552745$

Psychiat Prax 2015; 42:

353-355

(c) Georg Thieme Verlag KG

Stuttgart · New York

ISSN 0303-4259

Korrespondenzadresse

Prof. Dr. med.

Steffi G. Riedel-Heller, MPH

Institut für Sozialmedizin,

Arbeitsmedizin und Public

Health, Universität Leipzig,

Medizinische Fakultät

Philipp-Rosenthal-Straße 55

04103 Leipzig

Steffi.Riedel-Heller@medizin.

uni-leipzig.de

\section{Defizite bei der Versorgung schwer psychisch Kranker \\ $\nabla$}

Deutschlands Gesundheitssystem krankt an verschiedenen grundlegenden Problemen. Insbesondere drei Aspekte sind bei der Versorgung schwer psychisch Kranker relevant: (1) die Fragmentierung der Versorgung, die durch die unterschiedliche Vergütung ambulanter, stationärer und komplementärer Leistungen perpetuiert wird, (2) eine Diversifizierung des Systems, mit einer zunehmenden Anzahl von Akteuren und (3) der Umstand, dass ein belegtes Krankenhausbett besser finanziert wird als eine komplexe ambulante Behandlung [1]. Obgleich gemeindenah versorgt, profitieren gerade Menschen mit schweren psychischen Erkrankungen und einem komplexen Hilfebedarf in Deutschland nicht flächendeckend von evidenzbasierten Versorgungsangeboten, insbesondere von komplexen ambulanten Leistungen. Dazu zählen zum Beispiel die häusliche Akutbehandlung (Home Treatment) oder das sogenannte Assertive Community Treatment (ACT), eine längerfristige aufsuchende Behandlung im häuslichen Setting durch multiprofessionelle Teams [2, 3]. Der Anspruch auf ein Klinikbett, regelmäßige Facharztkonsultationen und der Zugang zu allen verfügbaren Psychopharmaka sind wichtig, aber nicht hinreichend für die Behandlung schwer psychisch Kranker [4]. Modellvorhaben zeigen, dass neue Finanzierungsformen die Etablierung neuer Versorgungsformen mit ambulanter Komplexbehandlung fördern und gestufte und kollaborative Hilfen möglich machen $[5,6]$. Gleichwohl hängt deren Etablierung oft von zufälligen lokalen Besonderheiten im Zusammenwirken verschiedener Akteure ab [4]. Die Weiterentwicklung der psychiatrisch-psychotherapeutischen Versorgung und die Schaffung der entsprechenden Rahmenbedingungen ist eine zentrale Aufgabe - bietet der Innovationsfonds hierfür Chancen?
Der Innovationsfonds: Initiierung, Finanzierung und allgemeine Zielbestimmung $\nabla$

Der Innovationsfonds ist Teil des GKV-Versorgungsstärkungsgesetzes ( $§ \S 92 \mathrm{a}$ und $92 \mathrm{~b}$ ), das am 23.7.2015 in Kraft getreten ist [7]. Mit dem Innovationsfonds sollen zukünftig in erster Linie innovative sektorenübergreifende Versorgungsformen, aber auch andere Versorgungsforschungsvorhaben gefördert werden. Dazu steht ab 2016 2019 eine jährliche Fördersumme von insgesamt 300 Mio. Euro zur Verfügung. 225 Mio. Euro sollen dezidiert in die Förderung neuer sektorübergreifender Versorgungsformen fließen (§92a, Abs. 1, SGB V). 75 Mio. Euro sollen in weitere Projekte der Versorgungsforschung investiert werden, die auf einen Erkenntnisgewinn zur Verbesserung der bestehenden Versorgung in der gesetzlichen Krankenversicherung ausgerichtet sind ( $§ 92 a$, Abs. 2, SGB V). Die Mittel werden durch den Gesundheitsfonds und die gesetzlichen Krankenkassen bereitgestellt. Dabei sollen insbesondere Vorhaben berücksichtigt werden, die über die bisherige Regelversorgung hinausgehen, einer besseren und sektorübergreifenden Versorgung verpflichtet sind und ein hinreichendes Potenzial aufweisen, dauerhaft in die Versorgung implementiert zu werden. Eine wissenschaftliche Begleitung ist zwingend. Ziel ist es, die Versorgungsqualität und Versorgungseffizienz zu verbessern, Versorgungsdefizite zu beheben und die Zusammenarbeit innerhalb und zwischen verschiedenen Versorgungsbereichen, Einrichtungen und Berufsgruppen zu optimieren. Vorrang haben interdisziplinäre und fachübergreifende Versorgungsmodelle, deren Erkenntnisse insbesondere auf andere Regionen und Indikationen übertragbar sind. Wichtig ist eine Verhältnismäßigkeit von Implementierungskosten und erwartbarem Nutzen $[8,9]$. 


\section{Was wird konkret gefördert? \\ $\nabla$}

Die Frage, welche innovativen Projekte der sektorübergreifenden Versorgung konkret mit diesen 225 Mio. Euro pro Jahr förderungswürdig sind, ist bisher unzureichend beantwortet [8]. In der Begründung zum GKV-Versorgungsstärkungsgesetz werden die Bereiche Telemedizin, Versorgungsmodelle in strukturschwachen Gebieten, Modelle mit Delegation und Substitution von Leistungen, Auf- und Ausbau der geriatrischen Versorgung, Modellprojekte zur Arzneimitteltherapiesicherheit bei multimorbiden Patienten und Einbeziehung von Möglichkeiten zur Verbesserung der Versorgungseffizienz von Patienten mit Migrationshintergrund genannt. Darüber hinaus wird darauf verwiesen, dass der einzurichtende Innovationsausschuss konkrete Förderschwerpunkte festlegt.

Die Versorgungsforschungsprojekte, für die jährlich 75 Mio. Euro zur Verfügung stehen, sollen auf einen Erkenntnisgewinn zur Verbesserung der bestehenden Versorgung der GKV ausgerichtet sein und insbesondere die Kriterien praktische Relevanz und besondere Nähe zur Patientenversorgung erfüllen. Die Projekte sollen Erkenntnisse liefern, die vom Gemeinsamen Bundesausschuss $(G-B A)$ in seine Richtlinien zur Gestaltung der Versorgung übernommen werden oder dem Gesetzgeber als Grundlage für strukturelle Veränderungen der gesetzlichen Grundlagen dienen können. Anträge können auch der wissenschaftlichen Begleitung und Auswertung von Verträgen nach $\S \S 73 c$ und 140a SGB V dienen, die vor Inkrafttreten des Gesetzes geschlossen wurden, sowie für Forschungsvorhaben zur Weiterentwicklung und insbesondere Evaluation der Richtlinien des Gemeinsamen Bundesausschusses eingesetzt werden. Auch hier wird eine Spezifizierung erwartet.

\section{Wer entscheidet?}

$\nabla$

Die Festlegung der Förderschwerpunkte, die Ausschreibungen und die Entscheidungen über die Förderanträge trifft der sogenannte Innovationsausschuss, der beim G-BA bis zum 1.Januar 2016 neu eingerichtet werden soll. Dem Innovationsausschuss gehören zehn stimmberechtigte Mitglieder an. Neben dem unparteiischen Vorsitzenden des G-BA finden sich Vertreter der gesetzlichen Krankenversicherung (GKV), der Kassenärztlichen Bundesvereinigung (KBV), der Kassenzahnärztlichen Bundesvereinigung (KZBV), der Deutschen Krankenhausgesellschaft (DKG), des Bundesministeriums für Gesundheit (BMG) und des Bundesministeriums für Bildung und Forschung (BMBF). Wo bleibt die Wissenschaft, wie werden die Versorgungsforscher in die Entscheidungen eingebunden? Ein Forschungsprogramm ohne Einbindung von Forschungsexpertise in die Entscheidungen erscheint widersinnig, so als ob man ein Krankenhaus ohne Ärzte betreiben möchte. Dieser Umstand war ein Hauptkritikpunkt am Referentenentwurf des Gesetzes, z.B. durch das Deutsche Netzwerk Versorgungsforschung (DNVF) und die Deutsche Gesellschaft für Psychiatrie, Psychotherapie, Psychosomatik und Nervenheilkunde (DGPPN) [10, 11]. Infolge der Diskussion wird dem Ausschuss nun ein Expertenbeirat zur Seite gestellt, der den Innovationsausschuss berät. In diesen Expertenbeirat werden vom Bundesministerium für Gesundheit bis zu zehn Vertreter aus Wissenschaft und Versorgungspraxis berufen.

\section{Noch viele Fragen sind offen \\ $\nabla$}

Diese kurze Beschreibung macht deutlich, dass in relativ kurzer Zeit ein weiter Weg zu gehen ist. Wie werden die Mittel des Innovationsfonds konkret vergeben? Welche Stimme hat die Wissenschaft? Wie wirken Expertenbeirat und Innovationsausschuss zusammen? Gelingt eine hochrangige Besetzung des Expertenbeirats? Kann die Arbeitsfähigkeit schnell hergestellt werden? Wie wird ein Begutachtungsverfahren für die Projekte initiiert? Wie werden Fachgutachter ausgewählt? Gelingt es, geeignete Evaluationskriterien für Projektanträge und Projekte aus dem Innovationsfonds zu implementieren, um die Mittelvergabe möglichst effektiv, effizient, gerecht, transparent und nachhaltig zu gestalten? Das Deutsche Netzwerk für Versorgungsforschung hat hier einen entsprechenden Vorschlag gemacht $[12,13]$. Ein weiterer offener Punkt ist der Umgang mit den Projektergebnissen. Welche Projekte werden für eine Übertragung in die Regelversorgung ausgewählt? Auch hier fehlen geeignete Verfahren, die alle Beteiligten, auch die Patienten, inkludieren. Möglicherweise werden einige dieser Fragen auf dem Deutschen Versorgungsforschungskongress in Berlin vom 7.-9. Oktober 2015 beantwortet.

\section{Chancen für die Versorgung psychisch Kranker und die psychiatrische Versorgungsforschung? \\ $\nabla$}

Die Bedeutung psychischer Erkrankungen für die Bevölkerungsgesundheit ist unumstritten. Die psychiatrisch-psychotherapeutische Versorgung war bei den ursprünglichen Projekten zur integrierten Versorgung, die mit einer Anschubfinanzierung gefördert wurden, unterrepräsentiert. Dies wurde vielfach beklagt. Der Innovationsfonds eröffnet nun zwei Wege, innovative Projekte für eine bessere und sektorübergreifende Versorgung psychisch Kranker zu starten. Zum einen können wissenschaftliche Einrichtungen (sofern sie den noch bekannt zu gebenden Förderschwerpunkten entsprechen) selbst ohne Krankenkassenpartnerschaft einen Antrag auf Förderung nach §92a, Abs.2, SGB V stellen. Viele der Themen der psychiatrischen Versorgungsforschung, die Experten in einem strukturierten Verfahren vor geraumer Zeit identifizierten, sind heute noch immer ganz oben auf der Agenda: (1) Versorgungswege und Anreize, (2) Prävention, (3) Ressourcenallokation (stationär vs. ambulant; leichte vs. schwere Störungen), (4) Barrieren für eine optimierte Versorgung aufseiten der Leistungserbringer und aufseiten der Nutzer, (5) Rolle von Komorbiditäten, (6) Nutzen von gestuften und kollaborativen Versorgungsansätzen, systematische Integration von Selbstmanagementelementen in die Regelversorgung (unter Nutzung neuer Medien), (7) Health Impact Assessments, (8) Benchmarking von Regionen: Identifizieren von Indikatoren für Qualität von Versorgungsangeboten, -systemen, -strukturen, -regionen [14]. Zum anderen können die in der Versorgungsforschung und in der Versorgungspraxis Tätigen aber die Chance ergreifen, selbst innovative Versorgungskonzepte zu entwickeln und Krankenkassen als Partner für diese Projekte zu gewinnen. Damit könnte auch eine Förderung von Projektideen über den Innovationsfond in einem Umfang erreicht werden, welcher der gesamtgesellschaftlichen Bedeutung des Fachgebiets angemessen ist.

Zeitiges Kommen sichert gute (Ausgangs)plätze. Es könnte sich lohnen, schon bei den ersten Ausschreibungen des Innovationsfonds mit dabei zu sein. Die psychiatrische Versorgungsforschung 
ist in Deutschland im Vergleich zu anderen Fachbereichen recht gut etabliert und dieses Programm bietet die Möglichkeit, weiter zur internationalen Entwicklung aufzuschließen [15]. Das gilt besonders auch für eine optimierte Versorgung schwer psychisch Kranker mit komplexen und langfristigen ambulanten Behandlungsbedarfen. Gerade für diese Personengruppe und deren Partizipation am Wissensfortschritt einer evidenzbasierten Versorgung trägt unsere Gesellschaft eine besondere Verantwortung. Der Innovationsfonds ist zweifelsohne ein ambitioniertes Projekt. Befürworter sprechen von „einem Quantensprung in der Entwicklung des Gesundheitswesens“. Die Initiative findet vor dem Hintergrund strukturkonservativer Rahmenbedingungen statt und es bleibt abzuwarten, ob die innovativen sektorübergreifenden Versorgungsmodelle nur ein „Verbindungspflaster“ für ein fragmentiertes Gesundheitssystem mit festgezurrten Finanzierungssäulen sind, oder ob diese Modelle den Beginn eines Durchbruchs für nachhaltige Verbesserungen markieren. Die Psychiatrische Praxis, das traditionelle Forum für psychiatrische Versorgungsforschung, wird diese Entwicklung intensiv und mit großer Spannung begleiten [16].

\section{Interessenkonflikt}

Die Autoren geben an, dass kein Interessenkonflikt besteht.

\section{Literatur}

1 Steinhart I, Wienberg G. Plädoyer für ein funktionales Basismodell gemeindepsychiatrischer Versorgung. Psychiat Prax 2014; 41: 179-181

2 Deutsche Gesellschaft für Psychiatrie, Psychotherapie und Nervenheilkunde (DGPPN), Hrsg. S3-Leitlinie Psychosoziale Therapien bei schweren psychischen Erkrankungen. S3-Praxisleitlinien in Psychiatrie und Psychotherapie. Berlin, Heidelberg: Springer; 2013

3 Riedel-Heller SG, Schomerus G, Gühne U et al. Schwer psychisch Kranke mit psychosozialen Therapien besser versorgen. Monitor Versorgungsforschung 2014; $7: 51-54$

4 Kilian R. Integrierte Versorgung unter gemeindepsychiatrischer Steuerung - Pro. Psychiat Prax 2014; 41: 182 - 183

5 Lambert M, Bock T, Daubmann A et al. Integrierte Versorgung von Patienten mit psychotischen Erkrankungen nach dem Hamburger Mo- dell: Teil 1. Rationalen, Behandlungsmodell und Ergebnisse der Vorstudie. Psychiat Prax 2014; 41: 257-265

6 Lambert M, Härter M, Brandes A et al. Transfer und Verstetigung von innovativen Aufklärungs- und Bildungsmaßnahmen, e-Mental Health und Versorgungsmodellen in psychenet - Hamburger Netz psychische Gesundheit. Psychiat Prax 2015; 42 (Suppl. 01): S80-88

7 Bundesministerium für Gesundheit. GKV-Versorgungsstärkungsgesetz. Im Internet: http://www.bmg.bund.de/themen/krankenversiche rung/gkv-versorgungsstaerkungsgesetz/gkv-vsg.html (Stand: 19.08. 2015)

8 Neugebauer EAM. Zum Innovationsfonds müssen wir eine intensive Diskussion führen! Monitor Versorgungsforschung 2015; 8: 27-29 (I-III)

9 Hecken J. Innovationsfonds - aus Sicht des G-BA. Im Internet: http:// www.netzwerk-versorgungsforschung.de/uploads/DNVF\%20Forum/ Hecken_GBA_Vortrag.pdf (Stand: 19.08.2015)

10 Deutsches Netzwerk Versorgungsforschung (DNVF) e.V. Stellungnahme des Deutschen Netzwerks Versorgungsforschung (DNVF) e.V. zum Gesetzentwurf der Bundesregierung vom 08.12.2014. Entwurf eines Gesetzes zur Stärkung der Versorgung in der gesetzlichen Krankenversicherung (GKV-Versorgungsstärkungsgesetz, GKV-VSG). Im Internet: http://www.netzwerk-versorgungsforschung.de/uploads/Stellungnah men/DNVF_Stellungnahme_GKV-VSG-Gesetzentwurf_150320.pdf (Stand: 19.08.2015)

11 Deutsche Gesellschaft für Psychiatrie, Psychotherapie und Nervenheilkunde (DGPPN). Entwurf des Versorgungsstärkungsgesetzes: DGPPN fordert umfassenderen Auftrag für eine strukturierte Versorgung bei psychischen Erkrankungen. Im Internet: https://www.dgppn.de/de/ presse/pressemitteilungen/detailansicht/article//entwurf-des.html (Stand: 19.08.2015)

12 Schmitt J, Petzold T, Pfaff $H$ et al. Innovationsfonds: Wohin mit den Fördermitteln? Dtsch Arztebl 2015; 112: A-1320 / B-1106 / C-1078

13 Schmitt J, Petzold T, Nellessen-Martens G et al. Priorisierung und Konsentierung von Begutachtungs-, Förder- und Evaluationskriterien für Projekte aus dem Innovationsfonds: Eine multiperspektivische Delphi-Studie. Gesundheitswesen 2015: Aug 13. [Epub ahead of print]

14 Bramesfeld A, Riedel-Heller S. Prioritäre Themen in der Versorgungsforschung zur psychischen Gesundheit. Psychiat Prax 2008; 35: 315-317

15 Riedel-Heller SG, König $H$. Methodische Grundlagen psychiatrischer Versorgungsforschung: aktueller Stand der Forschung und Entwicklung des Gebietes. In: Rössler W, Kawohl W, Hrsg. Soziale Psychiatrie. Das Handbuch für die psychosoziale Praxis; Bd. 1 Grundlagen. Stuttgart: Kohlhammer; 2013: 246-257

16 Riedel-Heller S, Bramesfeld A, Roick $C$ et al. Der Ruf nach mehr Versorgungsforschung. Psychiat Prax 2008; 35: 157-159 